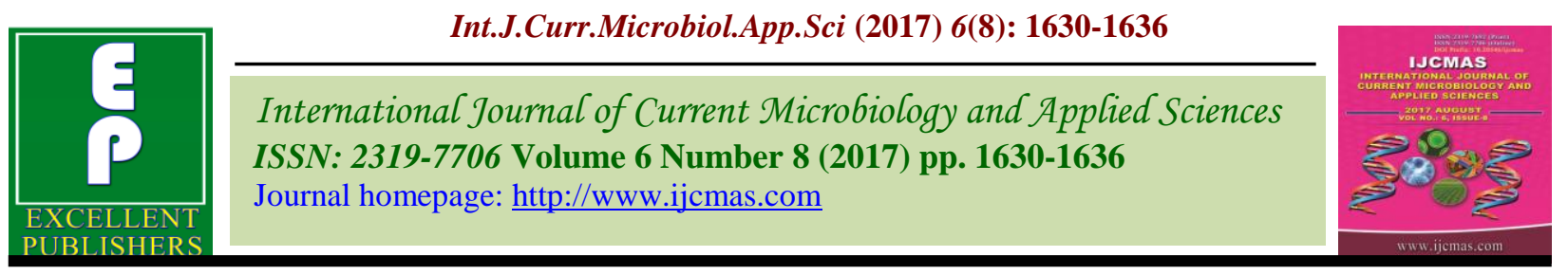

Original Research Article

https://doi.org/10.20546/ijcmas.2017.608.196

\title{
Effect of Integrated Weed Management on Yield, Quality and Economics of Summer Sorghum (Sorghum bicolor L.)
}

\author{
B.R. Verma*, H.M. Virdia and Dinesh Kumar \\ Department of Agronomy, N.M. College of Agriculture, Navsari Agricultural University, \\ Navsari - 396 450, Gujarat, India \\ *Corresponding author
}

A B S T R A C T

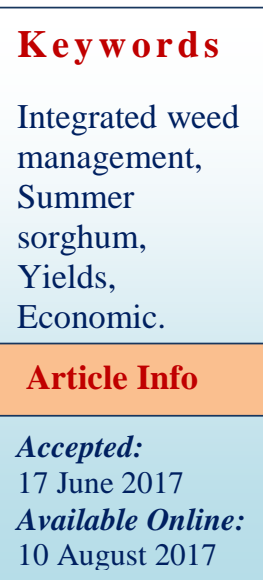

\section{Keywords}

Integrated weed

management

Summer

Economic.

Article Info

Accepted:

Available Online:

August 2017
A field experiment was conducted at College Farm, Navsari Agricultural University, Navsari (Gujarat) during summer season of the year 2016 under south Gujarat. The experiment was laid out in randomized block design with three replications and ten treatments with pre and post emergence herbicides (Atrazine, 2, 4-D and metsulfuron methyl) alone or combination of these herbicide followed by hand weeding and interculturing operation. Two hand weeding and inter-culturing operation at 20 and 40 DAS recorded significantly higher grain $(3302.67 \mathrm{~kg} / \mathrm{ha})$ and straw $(7638.67 \mathrm{~kg} / \mathrm{ha})$ yields. However, grain and straw yield was remained at par with treatments $\mathrm{T}_{8}$ i.e. Atrazine @ $0.50 \mathrm{~kg}$ /ha as PE $f b$ Atrazine @ $0.50 \mathrm{~kg} / \mathrm{ha}$ PoE 25 DAS $f b \mathrm{HW}$ and IC at 40 DAS (2936.67 kg/ha, $7374 \mathrm{~kg} / \mathrm{ha}$ ) and $\mathrm{T}_{9}$ i.e. Atrazine @ $0.50 \mathrm{~kg} / \mathrm{ha}$ as PE $f b$ 2, 4-D @ 0.50 $\mathrm{kg} / \mathrm{ha}$ as PoE at $25 \mathrm{DAS} f b \mathrm{HW}$ and IC at 40 DAS $(2867 \mathrm{~kg} / \mathrm{ha}, 7274 \mathrm{~kg} / \mathrm{ha})$ respectively. The maximum net returns ( $₹ 54,623$ per ha) was realized under the treatment of two hand weeding and inter-culturing operation at 20 and 40 DAS followed by treatments $\mathrm{T}_{4}$ i.e Atrazine @ $0.50 \mathrm{~kg} / \mathrm{ha}$ as PE $f b$ atrazine $0.50 \mathrm{~kg} / \mathrm{ha}$ as post emergence (PoE) at $25 \mathrm{DAS}(₹$ 50,179 ). However, the maximum gross returns ( $₹ 88,969$ per ha) was recorded with treatment $\mathrm{T}_{2}$ i.e. two hand weeding and inter-culturing operation at 20 and 40 DAS followed by $\mathrm{T}_{8}$ i.e. Atrazine @ $0.50 \mathrm{~kg} / \mathrm{ha}$ as PE $f b$ Atrazine @ $0.50 \mathrm{~kg} / \mathrm{ha}$ PoE $25 \mathrm{DAS} f b$ HW and IC at 40 DAS ( $₹ 80,855$ ). Whereas, the maximum B:C was recorded under the treatment $\mathrm{T}_{4}$ i.e. Atrazine @ $0.50 \mathrm{~kg} / \mathrm{ha}$ as $\mathrm{PE} f b$ atrazine $0.50 \mathrm{~kg} / \mathrm{ha}$ as post emergence (PoE) at 25 DAS (2.88).

\section{Introduction}

Sorghum (Sorghum bicolor L.) is one of the globally important cereal crop after wheat, maize, rice and barley. Sorghum is a unique crop among the major cereals and the staple food and fodder crop of the world's poor and most food-insecure populations, located primarily in the semi-arid tropics. Sorghum is grown on 43.81 million ha area in the world, producing about 65.42 million tonnes grain with an average yield of $1523 \mathrm{~kg} / \mathrm{ha}$. India and USA have largest share of global sorghum area, while the maximum production of sorghum occur in the USA. India presently produces about 5.54 million tonnes of sorghum grain from area of 6.16 million ha and productivity of $884 \mathrm{~kg} / \mathrm{ha}$ (Anon., 2016). The crop is primarily produced in Maharashtra, Andhra Pradesh, Gujarat and Karnataka. In Gujarat, sorghum occupies about 0.14 million ha area and annual 
production of 0.19 million tonnes with productivity of $1350 \mathrm{~kg} / \mathrm{ha}$ (Anonymous, 2016).

Sorghum is one of the important dry land crop grown in poor lands with minimum inputs and in dry conditions that tolerate to heat, salt and water-logging. Sorghum is a preferred in tropical, warm and semiarid regions of the world with high temperature and water stress with the threat of climate change looming large on the crop productivity, sorghum is hardy crop that play an important role in food, feed and fodder security in dry-land economy (Paterson et al., 2009). The integration of herbicides with some cultural operations and use of pre-emergence, post-emergence herbicides in combination with mechanical methods can prove to be more successful. This way integrated weed management is gaining importance in management of weeds for preventing losses and higher input-use efficiency (Ishya et al., 2007).

\section{Materials and Methods}

The experiment was conducted at plot number B-12 of the College Farm, N. M. College of Agriculture, Navsari Agricultural University, Navsari during summer season of 2016. Navsari Agricultural University campus is located on southern part of Gujarat state and geographically located at $20^{\circ} 57^{\prime} \mathrm{N}$ latitude and $72^{\circ} 54^{\prime} \mathrm{E}$ longitude at an altitude of 10 meters above the mean sea level. The soil of the experimental field was clayey in texture, dark grayish brown type, flat topography and characterized by medium to poor drainage with good water holding capacity. The soil available nutrient status showed lower availability of nitrogen $(195.16 \mathrm{~kg} / \mathrm{ha})$ medium for phosphorus $(29.83 \mathrm{~kg} / \mathrm{ha})$ and high for potassium $(364.70 \mathrm{~kg} / \mathrm{ha})$. The soil was slightly alkaline $\left(\mathrm{P}^{\mathrm{H}}\right.$ 7.7) with normal electric conductivity $(0.36 \mathrm{dS} / \mathrm{m})$. Ten treatments comprising of weed management practices viz., $\mathrm{T}_{1}$ : Un-weeded control, $\mathrm{T}_{2}$ :
Two hand weeding (HW) and inter-culturing at 20 and 40 DAS, T : Atrazine @ $1.0 \mathrm{~kg} / \mathrm{ha}$ as pre-emergence (PE), $\mathrm{T}_{4}$ : Atrazine @ 0.50 $\mathrm{kg} / \mathrm{ha}$ as PE $f b$ atrazine $0.50 \mathrm{~kg} / \mathrm{ha}$ as post emergence $(\mathrm{PoE})$ at $25 \mathrm{DAS}, \mathrm{T}_{5}$ : Atrazine @ $0.50 \mathrm{~kg} / \mathrm{ha}$ as PE $f b 2$, 4-D @ $0.50 \mathrm{~kg} / \mathrm{ha}$ as PoE at 25 DAS, T6: Atrazine @ 0.50 kg/ha as PE $f b$ metsulfuron methyl @ $6 \mathrm{~g} / \mathrm{ha}$ as PoE, $\mathrm{T}_{7}$ : Atrazine @ $1 \mathrm{~kg} / \mathrm{ha}$ as PE $f b \mathrm{HW}$ and IC at 40 DAS, T: Atrazine @ $0.50 \mathrm{~kg} / \mathrm{ha}$ as PE fb Atrazine @ $0.50 \mathrm{~kg} / \mathrm{ha}$ PoE 25 DAS fb HW and IC at 40 DAS, T9: Atrazine @ 0.50 $\mathrm{kg} / \mathrm{ha}$ as PE $f b 2,4-\mathrm{D} @ 0.50 \mathrm{~kg} / \mathrm{ha}$ as PoE at 25 DAS $f b$ HW and IC at 40 DAS, T 10 : Atrazine @ $0.50 \mathrm{~kg} / \mathrm{ha}$ as PE $f b$ metsulfuron methyl @6 g/ha as PoE 25 DAS fb HW and IC at 40 DAS, were evaluated in randomized block design with three replications. The improved and popular cultivar i.e. GJ 42 of sorghum was used for cultivation. The weed management treatments were used in sorghum with other cultural practices

\section{Results and Discussion}

\section{Effect on yield}

\section{Number of panicles per hill}

Significantly higher number of panicles (Table 1) in summer sorghum per hill was recorded under treatment $\mathrm{T}_{2}$ (2.60). While treatments $\mathrm{T}_{8}$ (2.50), $\mathrm{T}_{9}(2.40)$ and $\mathrm{T}_{7}$ (2.31) were found statistically at par with each other. Significantly higher number of panicles per hill was recorded due to lower weed competition in the treatments with good control in earlier days as well as in later phase of crop development. These results are in close conformity with Priya and Kubsad (2013).

\section{Seed yield}

Significantly higher seed yield (3302.67 $\mathrm{kg} / \mathrm{ha}$ ) (Table 1) were reported under 
treatment $\mathrm{T}_{2}$ and it was remain at par with treatments $\mathrm{T}_{8}(2936.67 \mathrm{~kg} / \mathrm{ha}), \mathrm{T}_{9} \quad(2867$ $\mathrm{kg} / \mathrm{ha})$ and $\mathrm{T}_{4}(2776.67 \mathrm{~kg} / \mathrm{ha})$. The higher seed yield was mainly due to maintenance of weed free environment, especially from initial as well as during critical growth stages of crop, reduce crop weed competition helped in better growth and development of sorghum crop resulting in more height and tillers which resulted in higher yield.

Table.1 Grain and straw yield of summer sorghum as influenced by different weed management practices

\begin{tabular}{|c|c|c|c|c|}
\hline \multirow{2}{*}{\multicolumn{2}{|c|}{ Treatment }} & \multirow{3}{*}{$\begin{array}{c}\begin{array}{c}\text { Number of } \\
\text { panicles } \\
\text { per hill }\end{array} \\
1.60\end{array}$} & \multirow{2}{*}{$\begin{array}{c}\text { Grain } \\
\text { yield (kg) } \\
\text { Per ha }\end{array}$} & \multirow{2}{*}{$\begin{array}{c}\begin{array}{c}\text { Straw yield } \\
\text { (kg) }\end{array} \\
\text { Per ha } \\
\end{array}$} \\
\hline & & & & \\
\hline $\mathrm{T}_{1}$ & Un weeded control & & 2164.00 & 5975.00 \\
\hline $\mathrm{T}_{2}$ & $\begin{array}{l}\text { Two hand weeding and inter- } \\
\text { culturing (IC) at } 20 \text { and } 40 \text { DAS }\end{array}$ & 2.60 & 3302.67 & 7638.67 \\
\hline $\mathrm{T}_{3}$ & $\begin{array}{l}\text { Atrazine @ } 1.0 \mathrm{~kg} / \mathrm{ha} \text { as pre- } \\
\text { emergence (PE) }\end{array}$ & 1.87 & 2577.67 & 6929.67 \\
\hline $\mathrm{T}_{4}$ & $\begin{array}{l}\text { Atrazine @ } 0.50 \mathrm{~kg} / \mathrm{ha} \text { as } \mathrm{PE} f b \\
\text { atrazine } 0.50 \mathrm{~kg} / \mathrm{ha} \text { as post } \\
\text { emergence }(\mathrm{PoE}) \text { at } 25 \mathrm{DAS}\end{array}$ & 2.13 & 2776.67 & 7134.00 \\
\hline $\mathrm{T}_{5}$ & $\begin{array}{l}\text { Atrazine @ } 0.50 \mathrm{~kg} / \mathrm{ha} \text { as PE } f b 2 \text {, } \\
\text { 4-D @ } 0.50 \mathrm{~kg} / \mathrm{ha} \text { as PoE at } 25 \\
\text { DAS }\end{array}$ & 2.00 & 2615.33 & 7003.33 \\
\hline $\mathrm{T}_{6}$ & $\begin{array}{l}\text { Atrazine @ } 0.50 \mathrm{~kg} / \mathrm{ha} \text { as } \mathrm{PE} f b \\
\text { metsulfuron methyl @ } 6 \mathrm{~g} / \mathrm{ha} \text { as } \\
\text { PoE }\end{array}$ & 1.70 & 2343.33 & 6396.00 \\
\hline $\mathrm{T}_{7}$ & $\begin{array}{l}\text { Atrazine @ } 1 \mathrm{~kg} / \mathrm{ha} \text { as PE } f b \text { hand } \\
\text { weeding and IC at } 40 \text { DAS }\end{array}$ & 2.31 & 2804.33 & 7161.67 \\
\hline $\mathrm{T}_{8}$ & $\begin{array}{l}\text { Atrazine @ } 0.50 \mathrm{~kg} / \mathrm{ha} \text { as PE } f b \\
\text { atrazine @ } 0.50 \mathrm{~kg} / \mathrm{ha} \text { PoE } 25 \\
\text { DAS } f b \text { hand weeding and IC at } 40 \\
\text { DAS }\end{array}$ & 2.50 & 2936.67 & 7374.00 \\
\hline $\mathrm{T}_{9}$ & $\begin{array}{l}\text { Atrazine @ } 0.50 \mathrm{~kg} / \mathrm{ha} \text { as PE } f b 2 \text {, } \\
\text { 4-D @ } 0.50 \mathrm{~kg} / \mathrm{ha} \text { as PoE at } 25 \\
\text { DAS } f b \mathrm{HW} \text { and IC at } 40 \text { DAS. }\end{array}$ & 2.40 & 2867.00 & 7242.00 \\
\hline $\mathrm{T}_{10}$ & $\begin{array}{l}\text { Atrazine @ } 0.50 \mathrm{~kg} / \mathrm{ha} \text { as } \mathrm{PE} f b \\
\text { metsulfuron methyl @ } 6 \mathrm{~g} / \mathrm{ha} \text { as } \\
\text { PoE 25 DAS } f b \mathrm{HW} \text { and IC at } 40 \\
\text { DAS }\end{array}$ & 1.90 & 2453.33 & 6512.33 \\
\hline & S.Em \pm & 0.14 & 187.58 & 318.36 \\
\hline & C.D. at $5 \%$ & 0.41 & 557.34 & 945.93 \\
\hline & C.V. & 11.39 & 12.10 & 7.95 \\
\hline
\end{tabular}


Table.2 Harvest index, test weight (g) and protein content in grain of summer sorghum as influenced by different weed management practices

\begin{tabular}{|c|c|c|c|c|}
\hline \multicolumn{2}{|r|}{ Treatment } & \multirow{2}{*}{$\begin{array}{c}\begin{array}{c}\text { Harvest } \\
\text { index }(\%)\end{array} \\
26.65\end{array}$} & \multirow{2}{*}{$\begin{array}{c}\begin{array}{c}\text { Test } \\
\text { weight }(\mathbf{g})\end{array} \\
21.73\end{array}$} & \multirow{2}{*}{$\begin{array}{c}\begin{array}{c}\text { Protein } \\
\text { content } \\
(\%)\end{array} \\
9.94\end{array}$} \\
\hline $\mathrm{T}_{1}$ & Un weeded control & & & \\
\hline $\mathrm{T}_{2}$ & $\begin{array}{l}\text { Two hand weeding and inter-culturing (IC) } \\
\text { at } 20 \text { and } 40 \text { DAS }\end{array}$ & 30.20 & 24.28 & 10.63 \\
\hline $\mathrm{T}_{3}$ & $\begin{array}{l}\text { Atrazine @ } 1.0 \mathrm{~kg} / \mathrm{ha} \text { as pre-emergence } \\
\text { (PE) }\end{array}$ & 28.21 & 23.45 & 10.19 \\
\hline $\mathrm{T}_{4}$ & $\begin{array}{l}\text { Atrazine @ } 0.50 \mathrm{~kg} / \mathrm{ha} \text { as PE } f b \text { atrazine } \\
0.50 \mathrm{~kg} / \mathrm{ha} \text { as post emergence (PoE) at } 25 \\
\text { DAS }\end{array}$ & 27.99 & 23.57 & 10.15 \\
\hline $\mathrm{T}_{5}$ & $\begin{array}{l}\text { Atrazine @ } 0.50 \mathrm{~kg} / \mathrm{ha} \text { as PE } f b \text { 2, 4-D @ } \\
0.50 \mathrm{~kg} / \mathrm{ha} \text { as PoE at } 25 \mathrm{DAS}\end{array}$ & 27.13 & 23.52 & 10.31 \\
\hline $\mathrm{T}_{6}$ & $\begin{array}{l}\text { Atrazine @ } 0.50 \quad \mathrm{~kg} / \mathrm{ha} \text { as } \mathrm{PE} \quad f b \\
\text { metsulfuron methyl @ } 6 \mathrm{~g} / \mathrm{ha} \text { as PoE }\end{array}$ & 26.93 & 22.95 & 10.25 \\
\hline $\mathrm{T}_{7}$ & $\begin{array}{l}\text { Atrazine @ } 1 \mathrm{~kg} / \mathrm{ha} \text { as PE } f b \mathrm{HW} \text { and IC at } \\
40 \mathrm{DAS}\end{array}$ & 27.01 & 23.85 & 10.35 \\
\hline $\mathrm{T}_{8}$ & $\begin{array}{l}\text { Atrazine @ } 0.50 \mathrm{~kg} / \mathrm{ha} \text { as PE } f b \text { atrazine @ } \\
0.50 \mathrm{~kg} / \mathrm{ha} \text { PoE } 25 \text { DAS } f b \mathrm{HW} \text { and IC at } \\
40 \mathrm{DAS}\end{array}$ & 28.53 & 24.00 & 10.44 \\
\hline $\mathrm{T}_{9}$ & $\begin{array}{l}\text { Atrazine @ } 0.50 \mathrm{~kg} / \mathrm{ha} \text { as PE } f b 2,4-\mathrm{D} @ \\
0.50 \mathrm{~kg} / \mathrm{ha} \text { as PoE at } 25 \mathrm{DAS} f b \mathrm{HW} \text { and } \\
\text { IC at } 40 \text { DAS }\end{array}$ & 28.40 & 23.89 & 10.36 \\
\hline $\mathrm{T}_{10}$ & $\begin{array}{l}\text { Atrazine @ } 0.50 \quad \mathrm{~kg} / \mathrm{ha} \text { as } \mathrm{PE} f b \\
\text { metsulfuron methyl } 6 \mathrm{~g} / \mathrm{ha} \text { as } \mathrm{PoE} 25 \\
\text { DAS } f b \mathrm{HW} \text { and IC at } 40 \mathrm{DAS}\end{array}$ & 27.41 & 23.33 & 10.26 \\
\hline & S.Em \pm & 1.76 & 0.41 & 0.11 \\
\hline & C.D. at $5 \%$ & NS & 1.21 & 0.32 \\
\hline & C.V. & 10.92 & 3.02 & $1 . .81$ \\
\hline
\end{tabular}


Table.3 Economics as influenced by different weed management practices of summer sorghum

\begin{tabular}{|c|c|c|c|c|}
\hline \multicolumn{2}{|r|}{ Treatment } & \multirow{2}{*}{$\begin{array}{c}\begin{array}{c}\text { Gross return } \\
(\text { ₹ per ha) }\end{array} \\
61205 \\
\end{array}$} & \multirow{2}{*}{$\begin{array}{c}\begin{array}{c}\text { Net return } \\
\text { ( } \text { ₹ per ha) }\end{array} \\
35559\end{array}$} & \multirow{2}{*}{$\begin{array}{r}\text { B:C } \\
\text { Ratio } \\
2.39\end{array}$} \\
\hline $\mathrm{T}_{1}$ & Un weeded control & & & \\
\hline $\mathrm{T}_{2}$ & $\begin{array}{l}\text { Two hand weeding and inter- } \\
\text { culturing (IC) at } 20 \text { and } 40 \text { DAS }\end{array}$ & 88969 & 54623 & 2.59 \\
\hline $\mathrm{T}_{3}$ & $\begin{array}{l}\text { Atrazine @ } 1.0 \mathrm{~kg} \text { /ha as pre- } \\
\text { emergence (PE) }\end{array}$ & 72342 & 45761 & 2.72 \\
\hline $\mathrm{T}_{4}$ & $\begin{array}{l}\text { Atrazine @ } 0.50 \mathrm{~kg} / \mathrm{ha} \text { as } \mathrm{PE} f b \\
\text { atrazine } 0.50 \mathrm{~kg} / \mathrm{ha} \text { as post } \\
\text { emergence }(\mathrm{PoE}) \text { at } 25 \mathrm{DAS}\end{array}$ & 76935 & 50179 & 2.88 \\
\hline $\mathrm{T}_{5}$ & $\begin{array}{l}\text { Atrazine @ } 0.50 \mathrm{~kg} / \mathrm{ha} \text { as PE } f b 2 \text {, } \\
0 \mathrm{~kg} / \mathrm{ha} \text { as } \mathrm{PoE} \text { at } 25 \mathrm{DAS}\end{array}$ & 73317 & 46741 & 2.76 \\
\hline $\mathrm{T}_{6}$ & $\begin{array}{l}\text { Atrazine @ } 0.50 \mathrm{~kg} / \mathrm{ha} \text { as } \mathrm{PE} f b \\
\text { metsulfuron methyl @ } 6 \mathrm{~g} / \mathrm{ha} \text { as } \\
\text { PoE }\end{array}$ & 66055 & 39229 & 2.46 \\
\hline $\mathrm{T}_{7}$ & $\begin{array}{l}\text { Atrazine @ } 1 \mathrm{~kg} / \mathrm{ha} \text { as PE } f b \mathrm{HW} \\
\text { and IC at } 40 \text { DAS }\end{array}$ & 77572 & 46641 & 2.51 \\
\hline $\mathrm{T}_{8}$ & $\begin{array}{l}\text { Atrazine @ } 0.50 \mathrm{~kg} / \mathrm{ha} \text { as PE } f b \\
\text { atrazine @ } 0.50 \mathrm{~kg} / \mathrm{ha} \text { PoE } 25 \mathrm{DAS} \\
\mathrm{fb} \mathrm{HW} \text { and IC at } 40 \mathrm{DAS}\end{array}$ & 80855 & 49749 & 2.60 \\
\hline $\mathrm{T}_{9}$ & $\begin{array}{l}\text { Atrazine @ } 0.50 \mathrm{~kg} / \mathrm{ha} \text { as PE } f b 2 \text {, } \\
4-\mathrm{D} @ 0.50 \mathrm{~kg} / \mathrm{ha} \text { as PoE at } 25 \\
\text { DAS } f b \mathrm{HW} \text { and IC at } 40 \mathrm{DAS}\end{array}$ & 79066 & 48140 & 2.56 \\
\hline $\mathrm{T}_{10}$ & $\begin{array}{l}\text { Atrazine @ } 0.50 \mathrm{~kg} / \mathrm{ha} \text { as PE } f b \\
\text { metsulfuron methyl @ } 6 \text { g/ha as } \\
\text { PoE 25 DAS } f b \mathrm{HW} \text { and IC at } 40 \\
\text { DAS }\end{array}$ & 68604 & 37428 & 2.20 \\
\hline
\end{tabular}

Seed yield is primarily a function of accumulation of photosynthates resulted in growth and increase yield. These results are in corroboration with findings of Dhar et al., (2006), Rathod et al., (2010), Kumar et al., (2012) and Priya and Kubsad (2013).

\section{Straw yield}

Significantly higher straw yield was reported under treatment $\mathrm{T}_{2}(7638.67 \mathrm{~kg} / \mathrm{ha})$ (Table 1) and it was remain at par with treatments $\mathrm{T}_{8}$ (7374 kg/ha), $\mathrm{T}_{9}(7274 \mathrm{~kg} / \mathrm{ha}), \mathrm{T}_{7}(7161.67$ $\mathrm{kg} / \mathrm{ha}), \mathrm{T}_{4}(7134 \mathrm{~kg} / \mathrm{ha}), \mathrm{T}_{5}(7003.33 \mathrm{~kg} / \mathrm{ha})$ and $T_{3}(6929.67 \mathrm{~kg} / \mathrm{ha})$. Significantly higher straw yield was revealed due to avoiding crop weed competition under these treatments with affect on growth and yield attributes. These results are in corroboration with finding of Dhar et al., (2006), Rao et al., (2007), Kumar et al., (2012) and Priya and Kubsad (2013).

\section{Harvest index}

It can be seen from the (Table 2) that treatment $\mathrm{T}_{2}(30.20 \%)$ had higher harvest index followed by treatments $\mathrm{T}_{8}(28.53 \%), \mathrm{T}_{9}$ $(28.40 \%)$ and $\mathrm{T}_{3}(28.21 \%)$. 


\section{Test weight}

Treatment $\mathrm{T}_{2}(24.28 \mathrm{~g})$ recorded significantly higher test weight and noticed that most of treatments (Table 2) were at par with it except treatments $\mathrm{T}_{1}(21.73 \mathrm{~g})$ and $\mathrm{T}_{6}(22.95 \mathrm{~g})$. Significantly higher test weight noticed due to lesser weed competition in treatments at earlier as well as later phase of crop growth period. Similar results were also reported by Priya and Kubsad (2013).

\section{Protein content in seed}

Treatment $T_{2}$ registered significantly higher protein content $(10.63 \%)$ which was at par with the treatments $\mathrm{T}_{8}(10.44 \%), \mathrm{T}_{9}(10.36$ $\%), \mathrm{T}_{7}(10.35 \%)$ and $\mathrm{T}_{5}(10.31 \%)$. While, significantly lower protein content was recorded under un-weeded control $(9.94 \%)$ over rest of treatments (Table 2).

This was fact that weed management controlled weed effectively and significantly reduction in crop-weed competition ultimately benefited to the crop and higher uptake and utilization of nutrients directly or indirectly that help in protein synthesis in grain of sorghum. Similar results were also reported by Dhar et al., (2006) and Rao et al., (2007).

\section{Effect on economics}

Treatment $\mathrm{T}_{2}$ recorded maximum gross return of (₹ 88,969 per ha) followed by treatments $\mathrm{T}_{8}$ ( $₹ 80,855$ per ha) $\mathrm{T}_{9}$ (₹ 79,066 per ha) and $\mathrm{T}_{7}$ (₹ 77,572 per ha) (Table 3). The higher grain yields (Table 2) recorded under these treatments might be responsible for higher gross return. Here the highest net return ( $₹$ 54,623 per ha) was recorded in treatment $T_{2}$ followed by treatment $\mathrm{T}_{4}$ ( $₹ 50,179$ per ha), $\mathrm{T}_{8}$ (₹49,749 per ha). Treatment $T_{1}$ registered the lowest net return of ( $₹ 35,559$ per ha) compare to these treatments, followed by treatments $T_{6}$ and $\mathrm{T}_{10}$ may be due to phytotoxic effect of metsulfuron methyl on these treatments as well as more competitive effect on growth and yield resulted in poor return.

Whereas, the maximum B:C was accrued under the treatment $\mathrm{T}_{4}(2.88)$ followed by $\mathrm{T}_{5}$ (2.76), $\mathrm{T}_{3}$ (2.72) and $\mathrm{T}_{8}(2.60)$. Higher gross returns along with the lowest cost under treatments $\mathrm{T}_{1}, \mathrm{~T}_{3}, \mathrm{~T}_{4}, \mathrm{~T}_{5}$ and $\mathrm{T}_{6}$ might be responsible for higher net return and $\mathrm{B}$ : $\mathrm{C}$ ratio. This is in conformity with the findings of Kumar et al., (2012), Mishra et al., (2012), Priya and Kubsad (2013), Patel et al., (2014) and Thakur et al., (2016).

\section{References}

Anonymous. 2016. Sorghum area, production and productivity. www.india stat.com

Dhar, S., Das, S.K., Kumar, S. and Thripathi, S. B. 2006. Response of fodder sorghum to different weed management techniques and nitrogen levels. Indian J. Agron., 51(4): 310-313.

Ishaya, D.B., Dadari, S.A. and Shebayan, J.A.Y. 2007. Evaluation of herbicides for weed control in sorghum (Sorghum bicolor) in Nigeria. Crop Protection, 26: 1697-1701.

Kumar, V., Tyagi, S. and Singh, D. 2012. Yield, $\mathrm{N}$ uptake and economics of fodder sorghum and associated weeds as affected by different weed management practices. Progressive Agri., 12(1): 96-102.

Mishra, J.S., Rao, S.S. and Dixit, A. 2012. Evaluation of new herbicides for weed control and crop safety in rainy season sorghum. Indian J. Weed Sci., 44(1): 71-72.

Patel, Z.N., Solanki, B.G., Jadhav, B.D., Patel, J.G., Triviedy, S.J., and Patel, J.R. 2014. Integrated weed management in kharif sorghum. In: Proceeding of 10th Combined Joint AGRESCO 
meeting, Natural Resource Management Sub committee, N.A.U., Navsari. pp. 96100.

Paterson, A.H., Bowers J.E. and Bruggmann, R. 2009. The Sorghum bicolor genome and the diversification of grasses. Nature, 457: 551-556.

Priya, H.R. and Kubsad, V.S. 2013. Integrated weed management in rainy season sorghum (Sorghum bicolor). Indian J. Agron., 58(4): 548-553.

Rao, S.S., Regar, P.L., Jangid, B.L. and Singh, Y.V. 2007. Effect of nutrient and weed management on forage sorghum
(Sorghum bicolor) under rainfed condition. Indian J. Agron., 52(2): 139142.

Rathod, S.S., Zade, K.K. and Jawale, S.A. 2010. Integrated weed management in kharif sorghum. Int. J. Forestry Crop Improvement, 1: 109-111.
Thakur, N.S., Kushwaha, B.B., Girothia, O.P., Sinha, N.K. and Mishra, J.S. 2016. Effect of integrated weed management on growth and yields of rainy season sorghum. Indian J. Agron., 61(2): 217222.

\section{How to cite this article:}

Verma, B.R., H.M. Virdia and Dinesh Kumar. 2017. Effect of Integrated Weed Management on Yield, Quality and Economics of Summer Sorghum (Sorghum bicolor L.). Int.J.Curr.Microbiol.App.Sci. 6(8): 1630-1636. doi: https://doi.org/10.20546/ijcmas.2017.608.196 